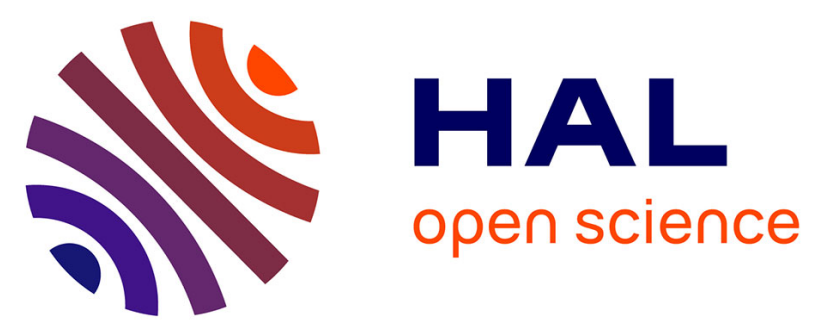

\title{
Hypolipidaemia among patients with PMM2-CDG is associated with low circulating PCSK9 levels: a case report followed by observational and experimental studies
}

Michael Chong, Grace Yoon, Delia Susan-Resiga, Ann Chamberland, David

Cheillan, Guillaume Paré, Nabil G Seidah

\section{To cite this version:}

Michael Chong, Grace Yoon, Delia Susan-Resiga, Ann Chamberland, David Cheillan, et al.. Hypolipidaemia among patients with PMM2-CDG is associated with low circulating PCSK9 levels: a case report followed by observational and experimental studies. Biochemical Genetics, 2020, 57 (1), pp.11-17. 10.1136/jmedgenet-2019-106102 . hal-02901741

\section{HAL Id: hal-02901741}

https://hal.science/hal-02901741

Submitted on 14 Sep 2021

HAL is a multi-disciplinary open access archive for the deposit and dissemination of scientific research documents, whether they are published or not. The documents may come from teaching and research institutions in France or abroad, or from public or private research centers.
L'archive ouverte pluridisciplinaire HAL, est destinée au dépôt et à la diffusion de documents scientifiques de niveau recherche, publiés ou non, émanant des établissements d'enseignement et de recherche français ou étrangers, des laboratoires publics ou privés. 


\title{
Hypolipidaemia among patients with PMM2-CDG is associated with low circulating PCSK9 levels: a case report followed by observational and experimental studies
}

\author{
Michael Chong, ${ }^{1,2}$ Grace Yoon, ${ }^{3,4}$ Delia Susan-Resiga, ${ }^{5}$ Ann Chamberland, ${ }^{5}$ \\ David Cheillan, ${ }^{6}$ Guillaume Paré, ${ }^{1,7}$ Nabil G Seidah (i) ${ }^{5}$
}

\begin{abstract}
Background Proprotein convertase subtilisin/kexin type 9 (PCSK9) inhibitors are novel therapeutics for reducing low-density lipoprotein cholesterol (LDLC). While serious side-effects have not been observed in short-term clinical trials, there remain concerns that long-term PCSK9 inhibition may cause neurocognitive side-effects. Methods and results An adult male with childhoodonset global developmental delay, cerebellar atrophy and severe hypolipidaemia underwent extensive biochemical and genetic investigations. Initial testing revealed low circulating PCSK9 levels and a common loss-of-function PCSK9 polymorphism, but these findings did not fully account for severe hypolipidaemia. Whole-exome sequencing was subsequently performed and identified two pathogenic phosphomannose mutase 2 (PMM2) variants (p.Arg141His and p.Pro69Ser) known to cause PMM2-associated congenital disorder of glycosylation (PMM2-CDG). A diagnosis of PMM2-CDG was consistent with the proband's neurological symptoms and severe hypolipidaemia. Given that PMM2-CDG is characterised by defective protein $\mathrm{N}$-glycosylation and that PCSK9 is a negative regulator of $L D L C$, we postulated that loss of PCSK9 N-glycosylation mediates hypolipidaemia among patients with PMM2-CDG. First, in an independent cohort of patients with PMM2-CDG $(\mathrm{N}=8)$, we verified that circulating PCSK9 levels were significantly lower in patients than controls $(p=0.0006)$. Second, we conducted in vitro experiments in hepatocyte-derived cells to evaluate the effects of PCSK9 N-glycosylation loss on $L D L$ receptor (LDLR) activity. Experimental results suggest that defective PCSK9 N-glycosylation reduces the ability of circulating PCSK9 to degrade LDLR.

Conclusion Life-long exposure to genetically lower PCSK9 per se is unlikely to cause neurocognitive impairment. Both observational and experimental findings suggest that hypolipidaemia in PMM2-CDG may be partially mediated by loss of PCSK9 N-glycosylation and/or its regulators.
\end{abstract}

\section{INTRODUCTION}

Proprotein convertase subtilisin/kexin type 9 (PCSK9) is an important regulator of cholesterol metabolism. ${ }^{1} 2$ Low-density lipoprotein (LDL) receptor (LDLR) removes LDL cholesterol (LDLc) from circulation, and PCSK9 inhibits this process by binding to the LDLR, which eventually leads to its degradation..$^{2-5}$ Several monoclonal antibodies against PCSK9 have been developed to lower LDLc. So far, clinical trials suggest that PCSK9 inhibitors reduce both LDLc and risk of cardiovascular events without other significant impact on events, including neurocognitive sequelae, myalgia, diabetes and liver damage. ${ }^{6}$ However, the average follow-up of these clinical trials has been less than 3 years, and thus, the long-term effects of PCSK9 inhibition remain unclear.

The concern regarding adverse effects of longterm PCSK9 inhibition, particularly relating to those affecting neurocognitive function has been raised previously. First, PCSK9 was initially characterised in a systematic screen for proteins associated with cerebellar neuron cell death and thus was originally dubbed 'Neuronal Apoptosis Regulated Convertase-1'. Second, in addition to neuronal apoptosis, PCSK9 is also a regulator of neuronal differentiation in mice. ${ }^{7}$ Third, PCSK9 is highly expressed in the cerebellum of adult humans. ${ }^{8} 9$ Altogether, it remains unknown whether prolonged PCSK9 inhibition could have adverse neurocognitive effects.

In this case report, we describe a patient with very low circulating PCSK9 levels and a striking neurological phenotype. To rule out a secondary genetic cause of the neurological phenotype beyond PCSK9 mutations, we conducted wholeexome sequencing (WES) of the index case. Unexpectedly, we discovered pathogenic compound heterozygous mutations in the PMM2 gene, which encodes phosphomannomutase 2 that is highly expressed in many tissues, including liver and brain. PMM2 deficiency results in congenital disorder of glycosylation type -1 a (PMM2-CDG) ${ }^{10}$ While a diagnosis of PMM2-CDG is in keeping with the neurological phenotype, notably, hypolipidaemia is a commonly reported feature among patients with PMM2-CDG, prevalent in $74 \%$ of such patients. ${ }^{11}$ To evaluate whether hypolipidaemia among patients with PMM2-CDG may be mediated by low PCSK9 activity, we pursued a series of follow-up investigations including the following: (i) measurement of circulating PCSK9 levels in an independent cohort of patients with PMM2-CDG and (ii) in vitro experiments to elucidate the effects 
of defective PCSK9 N-glycosylation on its ability to enhance LDLR degradation.

\section{METHODS}

\section{Genetic investigations in the index case}

Sanger sequencing of PCSK9 and its 12 exons was conducted at the Institut de Recherches Cliniques de Montréal for the proband, father and mother. WES of the proband and his father was performed at the Genetic and Molecular Epidemiology Laboratory in Hamilton, Ontario, Canada. Two different WES technologies were utilised. For the proband, WES was conducted on the Illumina Hiseq1500 ( $2 \times 100$ bp reads) using the TrueSeq Exome Capture kit, and for the father, WES was completed on the Ion Torrent Proton sequencer $(1 \times 125 \mathrm{bp}$ reads) using the Ampliseq Exome Enrichment kit. Raw sequence reads were aligned to the human genome reference sequence (version hg19), variant calling was performed and poor quality variants were filtered out. The specific bioinformatics pipeline applied for the aforementioned processes depended on the WES technology. Specifically, the Genome Analysis Tool Kit V.2.5.2 and the Torrent Variant Calling V.5.0.3 pipelines were applied to the proband and father, respectively. ${ }^{12}$

The program, Annovar, was used to annotate variant mutation effects based on RefGene transcripts. ${ }^{13}$ Only rare nonsynonymous mutations within intellectual disability-associated genes were considered. Rare mutations were defined as those having a minor allele frequency (MAF) less than 0.01 within both external and internal databases. External databases included the NHLBI GO Exome Sequencing Project (ESP6500), the 1000 Genomes $(1 \mathrm{KG})$ project and the Exome Aggregate Consortium, and the genome Aggregation Database (gnomAD). ${ }^{14-16}$ The internal database consisted of $\sim 1000$ exome sequences, which were run on both Ion Torrent and Illumina WES systems. A MAF threshold of 0.01 was applied within each major ethnic strata of external databases (Europeans, Africans, Latin Americans, East Asians, South Asians) such that if a variant was common (MAF $>0.01$ ) in even a single ethnic group, then it was excluded. Nonsynonymous mutations included missense, stopgain, stoploss, splicing and insertion/deletion mutations. A list of 1136 candidate genes associated with intellectual disability was compiled from Gilissen et $a l^{17}$ (2014).

\section{Measurement of circulating PCSK9 levels}

Serum PCSK9 levels were measured in the proband and his father using an in-house high sensitivity ELISA as reported. ${ }^{18} 19$ Using the same assay, PCSK9 levels were also measured in an independent cohort of patients with PMM2-CDG $(\mathrm{N}=8)$ and age-matched controls without PMM2-CDG $(\mathrm{N}=6)$. These individuals were recruited from Lyon, France at the Centre de Biologie et de Pathologie Est. A diagnosis of PMM2-CDG was confirmed through genetic testing and/or transferrin isoform analysis. Differences in the levels of PCSK9 and total cholesterol levels between patients with PMM2-CDG and controls were evaluated using the Student's t-test. Statistical analysis was performed in 'R' V.3.5.0.

\section{Experimental characterisation of defective PCSK9 $\mathrm{N}$-glycosylation on LDLR}

Naïve HepG2 cells and HepG2 cells lacking PCSK9 (PCSK9-KO; Seidah et al ATVB in revision) were cultured in Dulbecco's modified Eagle minimal essential medium supplemented with $10 \%(\mathrm{v} / \mathrm{v})$ fetal bovine serum (Invitrogen) and were maintained at $37^{\circ} \mathrm{C}$ under $5 \% \mathrm{CO}_{2} \cdot{ }^{20}$ Cells were seeded in poly-L-lysine
$(50 \mu \mathrm{g} / \mathrm{mL})$ coated 12 -well plates $\left(1.2 \times 10^{5}\right.$ cells/well). For the co-transfection experiments, 24 hours post-seeding, cells were transfected using jetPEI (PolyPlus) and a total of $2 \mu \mathrm{g}$ of cDNA $(1.2 \mu \mathrm{g}$ PCSK9 cDNA, $0.8 \mu \mathrm{g}$ LDLR cDNA), according to manufacturer's protocol, followed by replacement of the culturing medium by serum-free medium 24 hours post-transfection and incubation at $37^{\circ} \mathrm{C}$ for an additional 24 hours. For the media swap experiments, 24 hours post-seeding, cells were cultivated for 24 hours in serum-free medium followed by an additional 24 hours incubation with conditioned media from HEK293 cells expressing either wildtype (WT) or N533Q PCSK9, or empty vector (no PCSK9 control).

Following the incubation times specific to each experiment, cultured cells were washed twice with ice-cold phosphatebuffered saline and lysed on ice with ice-cold, non-denaturing cell lysis buffer (20 mM Tris- $\mathrm{HCl}, \mathrm{pH} 8,137 \mathrm{mM} \mathrm{NaCl}, 2 \mathrm{mM}$ disodium ethylenediaminetetraacetic acid ( $\mathrm{Na}_{2}$ EDTA), 1\% nonyl phenoxypolyethoxylethanol (NP-40), 10\% glycerol, 4\% protease inhibitor cocktail without EDTA) for $40 \mathrm{~min}$, with gentle rotation. Cell lysates were cleared by centrifuging for $12 \mathrm{~min}$ at $15000 \mathrm{x} \mathrm{g}$ at $4^{\circ} \mathrm{C}$ and the total protein content was measured using the Bio-Rad DC Protein assay. In total, $15-20 \mu \mathrm{g}$ of protein was separated on $8 \%$ Tris glycine sodium dodecyl sulfate polyacrylamide gel electrophoresis (SDS-PAGE) gels and transferred to a polyvinylidene fluoride membrane. Western blotting was performed for human LDLR (goat anti-human LDLR, 1:1000; AF2148; R\&D Systems), PMM2 (rabbit anti-PMM2, 1:1000; 10666-1-AP; Proteintech), glyceraldehyde 3-phosphate dehydrogenase (GAPDH) (rabbit anti-GAPDH, 1:2500; ab9485; Abcam) and for human PCSK9-V5 (mouse anti-V5, 1:5000; Invitrogen). After incubation with the appropriate secondary antibodies, the membranes were revealed using Clarity Western ECL Substrate (Bio-Rad), imaged with a GelDoc XR +instrument (Bio-Rad) and the bands of interest quantified using ImageLab 5.2.1 software (Bio-Rad).

\section{RESULTS}

\section{Case presentation}

A young male patient was seen at the Neurogenetics clinic at the Hospital for Sick Children, Toronto, Canada. He had a longstanding history of developmental delay, ataxia, cerebellar atrophy, and initially presented with global developmental delays as a very young child. He first sat at 2 years, crawled at 3 years and was not able to walk until the age of 4 years. He spoke his first words at 3 years, and was not able to speak in full sentences until he was 8 years old. On functional review, he had moderate dysarthria, but no swallowing difficulties. He had fine motor difficulties and needed assistance to cut food and feed himself, as well as dress. He had definite imbalance while walking, with several falls on a weekly basis. He also had occasional episodes of bladder incontinence. He was otherwise healthy and did not require any medications.

On physical examination, he had significant scoliosis, mild winging of the scapulae, widely spaced inverted nipples and pectus excavatum. Cranial nerve exam revealed end-gaze nystagmus bilaterally. Muscle tone was mildly hypotonic, muscle bulk and strength were completely normal. Cerebellar examination revealed multiple abnormalities including dysmetria, dysdiadochokinesis, irregular fast finger movements and toe tapping. Gait analysis revealed a wide-based ataxic gait and Romberg was positive. Deep tendon reflexes were $1+$ bilaterally and plantar responses flexor. Sensation was normal to light touch, pain and proprioception; however, there was decreased vibration sense. 
Table 1 Lipid profiles of the proband and his father

\begin{tabular}{lll}
\hline & Proband & Father \\
\hline Total cholesterol, mmol/L (percentile) & $2.54(<5)$ & $5.14(30)$ \\
$\mathrm{HDLc}, \mathrm{mmol} / \mathrm{L}$ (percentile) & $1.53(>95)$ & $0.81(7)$ \\
$\mathrm{LDLc}, \mathrm{mmol} / \mathrm{L}$ (percentile) & $0.76(<5)$ & $3.23(28)$ \\
\hline Triglycerides, $\mathrm{mmol} / \mathrm{L}$ (percentile) & $0.55(<5)$ & $2.39(44)$ \\
PCSK9, $\mathrm{ng} / \mathrm{mL}$ (percentile) & $49.0(<30)$ & $72.9(<50)$ \\
\hline
\end{tabular}

Cholesterol percentiles were derived using age-standardised and sex-standardised percentiles from the Mayo Clinic study.

HDLc, high-density lipoprotein cholesterol; LDLc, low-density lipoprotein cholesterol;

PCSK9, proprotein convertase subtilisin/kexin type 9.

MRI of the brain revealed cerebellar atrophy associated with increased T2 hyperintense signal of the cerebellum. Nerve conduction studies revealed decreased motor nerve conduction velocities and polyneuropathy. Serum albumin, alpha-fetoprotein, alanine aminotransferase (ALT), aspartate aminotransferase (AST), gammaglutamyl transferase (GGT), International Normalized Ratio (INR), and partial thromblastin time (PTT) were all normal. Lipid profiling (table 1) revealed the levels of total cholesterol (fifth percentile), high-density lipoprotein cholesterol ( $>95$ th percentile) LDLc $(<5$ th percentile), triglycerides ( $<5$ th percentile) and PCSK9 ( $<30$ th percentile).

The father of the proband was generally healthy and unremarkable with respect to neurological or motor deficits. He had a body mass index of 28.2 and a history of type 2 diabetes, which was diagnosed at 35 years. Like the proband, the father also had lower-than-average total cholesterol (30th percentile), LDLc (28th percentile), triglycerides (44th percentile) and PCSK9 $(<50$ percentile) (table 1$)$.

\section{Targeted sequencing of PCSK9 to identify genetic cause of low PCSK9 levels}

We hypothesised that low PCSK9 levels in the father and the proband might be explained by loss-of-function (LOF) variants within the PCSK9 gene. Indeed, Sanger sequencing of PCSK9 led to the identification of several established LOF PCSK9 variants in both the proband and the father (figure 1). The proband was heterozygous for one known LOF PCSK9 variant (p.10Leu insertion), whereas the father was heterozygous for two LOF PCSK9 variants (p.10Leu insertion and p.Arg46Leu). Other studies have demonstrated that heterozygosity for the p.Arg46Leu and p.10Leu insertion variants is associated with a $15 \%$, and $14.2 \%$ reduction in LDLc, respectively. ${ }^{21-23}$ Based on the aggregate genetic effect of LOF PCSK9 variants, the father was predicted to have had 29.2\% lower LDLc (11th percentile), and the proband was predicted to have had 14.2\% lower LDLc (28th percentile). The higher-than-expected LDLc in the father (28th vs estimated 11 th percentile) could not be attributed to the presence of the aforementioned cardiometabolic LOF risk factors. In addition, the lower observed versus estimated LDLc in the proband ( $<5$ th vs estimated 28 th percentile), the low levels of triglycerides ( $<5$ th percentile), as well as the neuromuscular syndrome in the proband, led us to hypothesise that the higher-than-expected LDLc in the father and lower LDLc in the proband could be due to protein-altering mutations in other genes beyond PCSK9.

\section{WES to identify secondary genetic causes}

WES was performed in the proband and his father to identify secondary genetic causes of hypolipidaemia and the neurological phenotype. Overall, 49270 variants were detected in the
PCSK9

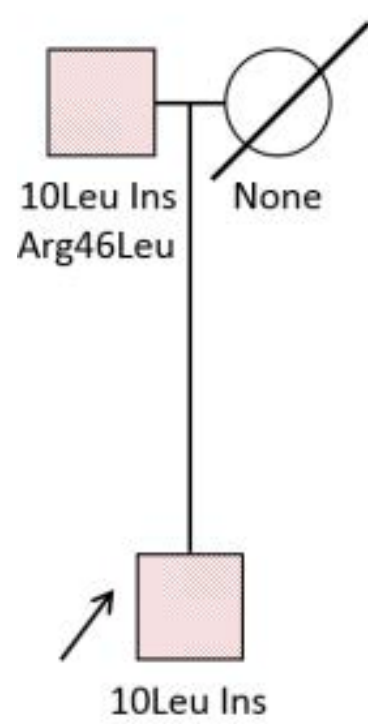

PMM2

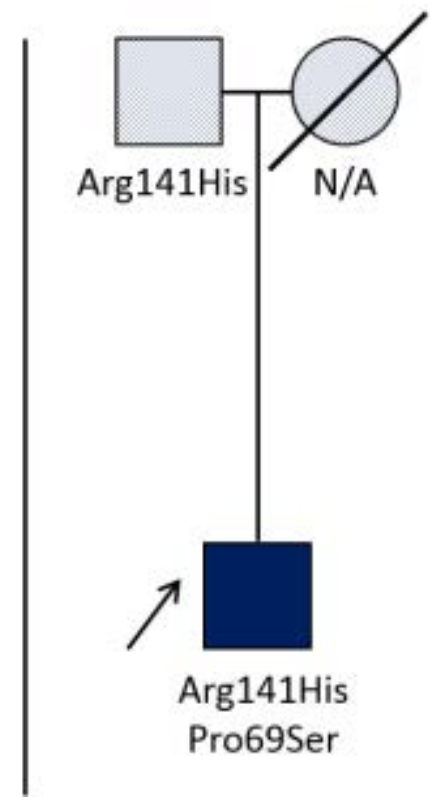

Figure 1 Pedigree summarising genetic findings. PCSK9 and PMM2 variants are displayed on the left and right, respectively. Variants are expressed in terms of their protein effects. Shapes with stippled patterns represent denote heterozygote carriers. PCSK9, proprotein convertase subtilisin/kexin type 9 .

proband. Several filters were applied to hone in on diseasecausing mutations. Given the severity of the disease, we reasoned that mutations were likely to be protein-altering and rare in the general population. Furthermore, because both the father and the mother did not exhibit neurological manifestations, diseasecausing mutations likely followed de novo or recessive inheritance in the proband (assuming complete penetrance). As such, the search for pathogenic variants was limited to those that were rare (MAF <0.01), non-synonymous, located within intellectual disability-associated genes, and compatible with de novo or recessive inheritance. After applying these criteria, 15 variants remained.

Among the 15 candidate variants were two heterozygous PMM2 variants, p.Arg141His and p.Pro69Ser (figure 1; table 2). Biallelic mutations in PMM2 cause CDG Type 1a (PMM2-CDG) and this specific configuration of compound heterozygous alleles has been described in a French family with PMM2-CDG. ${ }^{10} 24$ Furthermore, high-performance liquid chromatography assay revealed elevated disialo-transferrin and slightly decreased tetrasialo-transferrin, a pattern consistent with diagnosis of PMM2-CDG. Within the proband, p.Arg141His and p.Pro69Ser are likely situated in trans on different alleles. The father was heterozygous for the p.Arg141His allele but homozygous WT for Pro69. This suggests that p.Arg141His was transmitted to the proband paternally and p.Pro69Ser maternally. Unfortunately, it was not possible to directly confirm maternal inheritance of p.Pro69Ser as the mother was deceased. The variant p.Arg141His is the most frequently identified pathogenic mutation in patients with CDG, accounting for more than half of all PMM2-CDG cases. ${ }^{11}$ The frequency of the p.Arg141His variant in the gnomAD, the largest public repository of exome sequences, is 0.0041. In contrast, P69S was not observed in more than 120000 gnomAD participants (table 2). 


\begin{tabular}{|c|c|c|c|}
\hline Genetic variant & cDNA change & Protein change & $\begin{array}{l}\text { genome } \\
\text { Aggregation } \\
\text { Database } \\
\text { minor allele } \\
\text { frequency }\end{array}$ \\
\hline chr16:8905010 (G/A) & c.G422A & p.Arg141His & 0.0041 \\
\hline chr16:8898650 (C/T) & c.C205T & p.Pro69Ser & Not reported \\
\hline
\end{tabular}

The proband was heterozygous for both variants. CDNA and protein effects are reported according to the NM_000303 mRNA transcript.

\section{Circulating PCSK9 levels in additional patients with PMM2- CDG}

Circulating serum PCSK9 and total cholesterol levels were measured in an independent cohort of patients with PMM2-CDG $(\mathrm{N}=8)$ and age-matched controls $(\mathrm{N}=6)$. PCSK9 levels were $42 \%$ lower in patients with PMM2-CDG as compared with controls (mean difference $=-41.33 \mathrm{ng} / \mathrm{mL} ; 95 \%$ CI: -60.70 to $-21.97 ; p=0.0006$ ) (figure 2). Total cholesterol levels were 33\% lower in patients with PMM2-CDG as compared with controls (mean difference $=-1.33 \mathrm{mmol} / \mathrm{L} ; 95 \% \mathrm{CI}$ : -2.49 to -0.16 ; $\mathrm{p}=0.03$ ). Detailed characteristics of patients with PMM2-CDG are presented in online supplementary table S1.

\section{Experimental characterisation of defective PCSK9 $\mathrm{N}$-glycosylation on LDLR}

PCSK9 enhances the degradation of LDLR by two pathways: (i) a major extracellular pathway where circulating PCSK9 binds the cell surface LDLR and the endocytosed PCSK9-LDLR complex is targeted for lysosomal degradation, and (ii) a minor intracellular pathway by directly targeting the PCSK9-LDLR complex for degradation after its exit from the Golgi apparatus, best seen in transfection experiments.$^{25-27}$ PMM2-CDG is characterised by a global defect in $\mathrm{N}$-glycosylation affecting numerous proteins beyond PCSK9. To study whether the specific loss of PCSK9 N-glycosylation could account for hypolipidaemia among patients with PMM2-CDG, as an experimental model we used a PCSK9 p.Asn533Gln (N533Q) mutant, which abrogates the only PCSK9 N-glycosylation site at $\mathrm{Asn}_{533^{\circ}}{ }^{\text {. }}$

To study the functional consequences of the loss of PCSK9 $\mathrm{N}$-glycosylation on LDLR degradation, we selected two human hepatic HepG2 cell lines: naïve HepG2 cells as previously
A

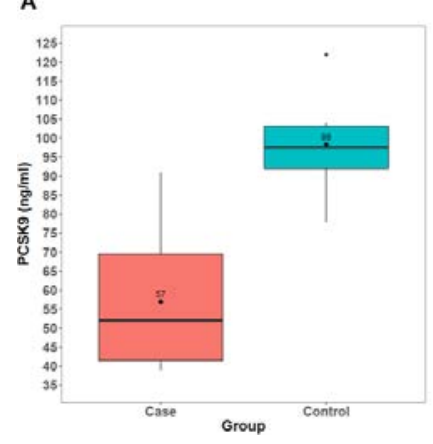

B

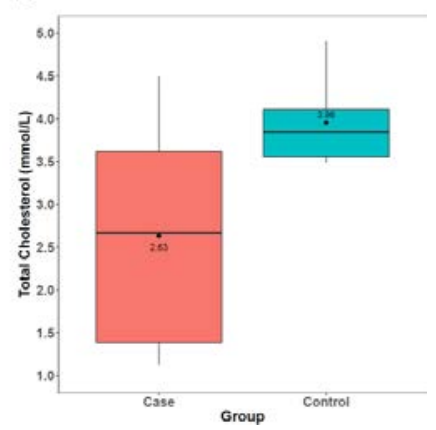

Figure 2 Comparison of circulating (A) PCSK9 and (B) total cholesterol levels between PMM2-congenital disorder of glycosylation patients $(N=8)$ and controls $(\mathrm{N}=6)$. The midline of each boxplot represent median values. The diamond represents mean values. Differences between groups were statistically significant for both circulating levels of PCSK9 $(p=0.0006)$ and total cholesterol $(p=0.03)$. PCSK9, proprotein convertase subtilisin/kexin type 9.
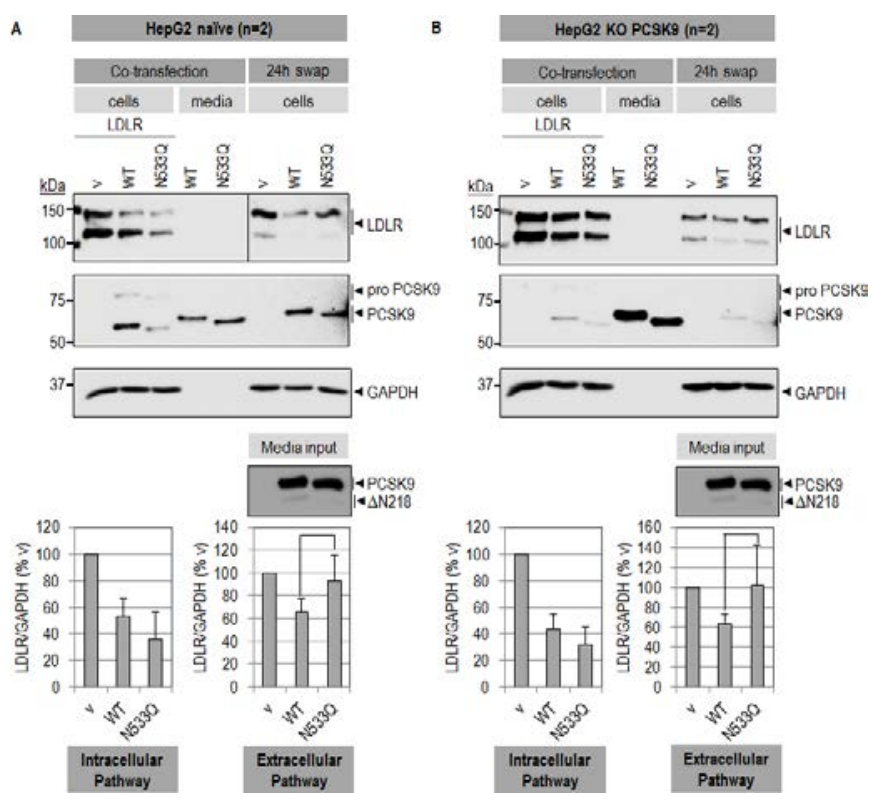

Figure 3 Effects of PCSK9 on LDLR degradation. ( $A$, intracellular pathway) HepG2 naive cells or (B, intracellular pathway) HepG2 PCSK9 $\mathrm{KO}$ cells were co-transfected with human LDLR and V5-tagged human PCSK9-WT (WT), PCSK9-N533Q (N533Q) or empty vector (v), as control. ( $A$, extracellular pathway) HepG2 naive cells or ( $B$, extracellular pathway) HepG2 PCSK9 KO cells were incubated for 24 hours with conditioned media from HEK293 cells (Media input): no PCSK9 media (v) or PCSK9media $(\sim 0.5 \mu \mathrm{g} / \mathrm{mL})$, WT or N533Q. Cell lysates were analysed by Western blot using a goat anti-human LDLR, mouse anti-V5 for PCSK9 and rabbit anti-GAPDH for loading control. Media were immunoblotted with mouse anti-V5 for PCSK9. The $\triangle \mathrm{N} 218$ is the Furin-cleaved form of PCSK9 at $\operatorname{Arg}_{218} \downarrow .^{26}$ Data are representative of three independent experiments, each performed in duplicate. Bars are averages \pm SD, and statistical analyses were performed by a Student's t-test. PCSK9, proprotein convertase subtilisin/ kexin type 9; KO, knockout; LDLR, low-density lipoprotein; WT, wild type.

reported $^{28}$ and HepG2 cells where PCSK9 was deleted by CRISPR-Cas9-mediated (HepG2-PCSK9) knockout (KO) of its gene (Seidah et al, in revision). These cells were analysed for PCSK9 activity following their co-transfection with human cDNAs coding for LDLR with C-terminally V5-tagged WT PCSK9 or its N533Q mutant (intracellular pathway), or on their incubation with media obtained from HEK293 cells that express either WT or N533Q PCSK9 (media swap; extracellular pathway), as reported. ${ }^{28}$

Western blot analyses demonstrate that in both cell lines (HepG2 naive and HepG2 KO PCSK9), loss of PCSK9 N-glycosylation (N533Q) does not significantly affect cellular LDLR levels via the intracellular pathway when compared with WT (co-transfection experiments) (figure 3A,B). However, a trend towards higher LDLR levels (loss of PCSK9 activity) was observed when the N533Q mutant was incubated with both cell lines as compared with WT (extracellular pathway; media swap) (figure $3 \mathrm{~A}, \mathrm{~B}$ ).

\section{Effect of PMM2 knockdown on PCSK9 and LDLR levels}

We next investigated the functional consequences of the knockdown of PMM2 on the N-glycosylation of PCSK9 and LDLR. Accordingly, we transfected PMM2 small interfering ribonucleic acid (siRNA) and control siRNA into naïve HepG2 cells and HepG2 cells stably expressing human PCSK9-V5 (at $\sim 150 \mathrm{ng} /$ $\mathrm{mL}$ levels, similar to human plasma levels). The data show that 
at 2 and $5 \mathrm{nM}$ siRNA, the best knockdown we could get was not more than $45 \%$ decrease in PMM2 mRNA levels with no significant changes in those of PCSK9, LDLR or sterol regulatory element-binding protein 2 (SREBP-2) (online supplementary figure S1). At the protein level, we also observed a decrease in PMM 2 by $\sim 40 \%$ at the $5 \mathrm{nM}$ siRNA concentration (online supplementary figure S2). At higher siRNA concentrations $(\geq 10 \mathrm{nM})$, cellular toxicity was observed based on visible cell rounding (not shown). Based on SDS-PAGE migration (as seen for PCSK9-N533Q, figure 3), 40\% of PMM2 knockdown did not affect the N-glycosylation of PCSK9 (cellular or secreted; $1 \mathrm{~N}$-glycosylation site) or cellular LDLR (4 N-glycosylation sites) (online supplementary figure S2). Since PMM2 could affect a number of proteins implicated in cellular trafficking, we noticed that under these knockdown conditions in HepG2 stable PCSK9-V5 cells, the levels of secreted PCSK9 were modestly, but significantly, reduced by $\sim 20 \%$ without affecting cellular LDLR levels (online supplementary figure S2). These results reveal that more than $\sim 50 \%$ reduction in PMM2 levels are needed to see any effect on $\mathrm{N}$-glycosylation of PCSK9 or LDLR, befitting the mild phenotype observed in individuals exhibiting heterozygote PMM2 mutations. Indeed, the father presented PCSK9 and LDLc levels that are close to normal, as opposed to the proband that exhibited $\sim 1.5$ and $\sim 4$ fold lower circulating PCSK9 and LDLc levels, respectively (table 1).

\section{DISCUSSION}

The proband presented with severe neurological deficits, hypolipidaemia and low circulating PCSK9 levels. Despite having lower LDLc levels, PCSK9 gene sequencing revealed fewer LOF variants in the proband than his father. Subsequent WES of all protein-coding genes led to the identification of two pathogenic variants within the PMM2 gene, leading to a diagnosis of PMM2-CDG. Accordingly, we explored whether defective PCSK9 N-glycosylation secondary to PMM2-CDG could account for the lower-than-expected LDLc levels in the proband. Indeed, we verified that circulating PCSK9 and total cholesterol levels were significantly lower in an independent cohort of patients with PMM2-CDG as compared with controls (online supplementary table S1). Furthermore, in vitro experiments suggested that loss of extracellular PCSK9 N-glycosylation may reduce its activity on cell surface LDLR (figure 3), thereby enhancing LDLR levels and resulting in lower circulating LDLc levels (hypolipidaemia).

PMM2-CDG is a CDG resulting in defective synthesis of $\mathrm{N}$-linked oligosaccharides, and hypolipidaemia is prevalent in approximately three out of every four patients. ${ }^{11}$ Hitherto, the mechanisms underlying hypolipidaemia in patients with PMM2-CDG have never been explored. Human PCSK9 has only one N-glycosylation site at $\mathrm{Asn}_{533}$, and the PCSK9 p.Asn533Gln (N533Q) mutant that would abrogate the N-glycosylation of PCSK9 does not hamper the folding or secretion of PCSK9 in human embryonic kidney cells (HEK293). ${ }^{7}$ In contrast, our experiments in human hepatocyte-derived cells (HepG2) suggest that defective $\mathrm{N}$-glycosylation may reduce its ability to enhance LDLR degradation via the extracellular but not the intracellular pathway. Since most PCSK9 function in the liver is mediated through the extracellular pathway, ${ }^{29}$ loss of PCSK9 N-glycosylation would be expected to result in overall reduced PCSK9 activity, higher LDLR levels and lower circulating LDLc. These experimental findings are concordant with the expectation that the proband with non-glycosylated PCSK9 had lower circulating LDLc levels than his heterozygote father.
Experimental results suggest that the specific loss of N-glycosylation on PCSK9 increases LDLR levels through a LOF mechanism of PCSK9 on LDLR. Furthermore, siRNA against PMM2 (40\% reduction) also resulted in a modest but significant lower secretion of PCSK9. Thus, absence of PMM2 and loss of PCSK9 N-glycosylation would together result in reduced secretion and PCSK9 activity on LDLR, leading to lower LDLc. This agrees with the observation that serum PCSK9 and LDLc levels were lower in patients with PMM2-CDG than matched controls. Furthermore, N-glycosylation defects of other proteins regulating PCSK9 secretion and/or activity may also contribute to hypolipidaemia. A limitation of our study is that we could not experimentally study the effects of severe PMM2 deficiency observed in classic PMM2-CDG cases, who retain 0\%-10\% of normal enzymatic activity. ${ }^{30}$ At more than $40 \%$ siRNA knockdown of PMM2 in human hepatocytes (ie, 60\% residual activity), cell toxicity was observed thus precluding a measurable effect on N-glycosylation. The latter level of PMM2 reduction is closer to the 50\%-70\% decrease in PMM2 activity observed in milder CDG cases and does not recapitulate levels of classic CDG. ${ }^{31}$ Altogether, our experimental and epidemiological findings suggest that hypolipidaemia in patients with PMM2-CDG may be mediated in part through defective N-glycosylation of PCSK9 and/or its regulators.

PCSK9 inhibition has become part of standard clinical treatment for patients with statin-resistant hyperlipidaemia or very high risk of atherosclerotic cardiovascular disease, but longterm side-effects remain unknown. Our original hypothesis that PCSK9 LOF variants explained the developmental delay in the proband was negated, as a secondary genetic cause (PMM2-CDG) befitting the neurological phenotype was discovered. Indeed, this is consistent with a recent phenome-wide analysis of the PCSK9 LOF variant, p.Arg46Leu, which found no association between mutation carrier status or PCSK9 inhibition and risk for cognitive dysfunction. ${ }^{32} 33$ Furthermore, while complete loss of PCSK9 in humans is rare, there are several documented cases in which these so-called 'human PCSK9 KOs' exhibit no conspicuous neurological defects so far. ${ }^{3435}$ Consistent with other genetic studies, this case report provides further reassurance that long-term PCSK9 inhibition or silencing is unlikely to cause neurocognitive decline.

Of note, several studies have shown that $\mathrm{N}$-glycans are important for the functional activity of amyloid precursor protein as well as its processing enzyme $\beta$-secretase, and defects in N-glycosylation have been linked to the development of Alzheimer's disease ${ }^{36} 37$ and other neurocognitive disorders such as those associated with hippocampal atrophy. ${ }^{38}$ Therefore, the LOF double heterozygote p.Arg141His and p.Pro69Ser PMM2 mutations found in the proband should result in defects in N-glycosylation of a variety of proteins, which in sum could affect many pathways including neurocognitive functions that are particularly sensitive to disturbances in protein posttranslational modifications. ${ }^{39}$

\footnotetext{
Author affiliations

${ }^{1}$ Genetic \& Molecular Epidemiology Laboratory, Population Health Research Institute, David Braley Cardiac Vascular and Stroke Research Institute, Hamilton Health Sciences, Hamilton, Ontario, Canada

${ }^{2}$ Department of Biochemistry \& Biomedical Sciences, McMaster University Faculty of Health Sciences, Hamilton, Ontario, Canada

${ }^{3}$ Department of Neurology and Clinical and Metabolic Genetics, Hospital for Sick Children, Toronto, Ontario, Canada

${ }^{4}$ Department of Paediatrics, University of Toronto, Toronto, Ontario, Canada ${ }^{5}$ Department of Biochemical Neuroendocrinology, Clinical Research Institute of Montreal, Montreal, Quebec, Canada
} 
${ }^{6}$ Service de Biochimie et Biologie Moléculaire Grand Est, Unité Médicale Pathologies Métaboliques, Erythrocytaires et Dépistage Périnatal, Centre de Biologie et de Pathologie Est, Hospices Civils de Lyon, Lyon, France

${ }^{7}$ Department of Pathology and Molecular Medicine, McMaster University Faculty of Health Sciences, Hamilton, Ontario, Canada

Correction notice This article has been corrected since it was published Online First. On page 3, two errors related to Pro69 and P69S have been corrected.

Acknowledgements We thank the index family, PMM2-CDG cases, and all matched controls, for participating in this study.

Contributors MC and GP analysed and interpreted exome sequencing results and performed all statistical analyses. GY recruited the index case and performed the clinical evaluation of the patient and his father. NS and DSR conducted all in vitro experiments. AC measured circulating PCSK9 levels in PMM2-CDG patients and the index cases' father. DC provided the secondary cohort of PMM2-CDG cases. MC, NS, and GY were major contributors in writing the manuscript. All authors read and approved the final manuscript. GP and NS are co-last authors.

Funding This work was supported by the Canadian Institutes of Health Research grants Foundation Scheme 148363, a Canada Research Chair 231335, and a Fondation Leducq grant \#13CVD03.

Competing interests GP has received consulting fees from Sanofi, Bristol-Myers Squibb, Lexicomp and Amgen and has received support for research through his institution from Sanofi. MC, GY, DSR, AC, DC and NS declare no competing interests.

Patient consent for publication Obtained.

Ethics approval This study was approved by the Research Ethics Board of the Hospital for Sick Children, REB \#1000009004.

Provenance and peer review Not commissioned; externally peer reviewed.

Data availability statement All data relevant to the study are included in the article or uploaded as supplementary information.

ORCID iD

Nabil G Seidah http://orcid.org/0000-0001-6503-9342

\section{REFERENCES}

1 Seidah NG. The PCSK9 revolution and the potential of PCSK9-based therapies to reduce LDL-cholesterol. Gcsp 2017;2017:e201702.

2 Seidah NG, Abifadel M, Prost S, Boileau C, Prat A. The proprotein convertases in hypercholesterolemia and cardiovascular diseases: emphasis on proprotein convertase subtilisin/kexin 9. Pharmacol Rev 2017;69:33-52.

3 Maxwell KN, Breslow JL. Adenoviral-Mediated expression of PCSK9 in mice results in a low-density lipoprotein receptor knockout phenotype. Proc Natl Acad Sci U S A 2004;101:7100-5.

4 Nassoury N, Blasiole DA, Tebon Oler A, Benjannet S, Hamelin J, Poupon V, McPherson PS, Attie AD, Prat A, Seidah NG. The cellular trafficking of the secretory proprotein convertase PCSK9 and its dependence on the LDLR. Traffic 2007;8:718-32.

5 Zhang D-W, Lagace TA, Garuti R, Zhao Z, McDonald M, Horton JD, Cohen JC, Hobbs $H H$. Binding of proprotein convertase subtilisin/kexin type 9 to epidermal growth factor-like repeat a of low density lipoprotein receptor decreases receptor recycling and increases degradation. J Biol Chem 2007;282:18602-12.

6 Karatasakis A, Danek BA, Karacsonyi J, Rangan BV, Roesle MK, Knickelbine T, Miedema MD, Khalili H, Ahmad Z, Abdullah S, Banerjee S, Brilakis ES. Effect of PCSK9 inhibitors on clinical outcomes in patients with hypercholesterolemia: a metaanalysis of 35 randomized controlled trials. J Am Heart Assoc 2017;6. doi:10.1161/ JAHA.117.006910. [Epub ahead of print: 09 Dec 2017]

7 Seidah NG, Benjannet S, Wickham L, Marcinkiewicz J, Jasmin SB, Stifani S, Basak A, Prat $A$, Chretien M. The secretory proprotein convertase neural apoptosis-regulated convertase 1 (NARC-1): liver regeneration and neuronal differentiation. Proc Natl Acad Sci U S A 2003:100:928-33.

8 Lonsdale J, Thomas J, Salvatore M, Phillips R, Lo E, Shad S, Hasz R, Walters G, Garcia F, Young N, Foster B, Moser M, Karasik E, Gillard B, Ramsey K, Sullivan S, Bridge J, Magazine H, Syron J, Fleming J, Siminoff L, Traino H, Mosavel M, Barker L, Jewell S, Rohrer D, Maxim D, Filkins D, Harbach P, Cortadillo E, Berghuis B, Turner L, Hudson E, Feenstra K, Sobin L, Robb J, Branton P, Korzeniewski G, Shive C, Tabor D, Qi L, Groch K, Nampally S, Buia S, Zimmerman A, Smith A, Burges R, Robinson K, Valentino K, Bradbury D, Cosentino M, Diaz-Mayoral N, Kennedy M, Engel T, Williams P, Erickson K, Ardlie K, Winckler W, Getz G, DeLuca D, MacArthur D, Kellis M, Thomson A, Young T, Gelfand E, Donovan M, Meng Y, Grant G, Mash D, Marcus Y, Basile M, Liu J, Zhu J, Tu Z, Cox NJ, Nicolae DL, Gamazon ER, Im HK, Konkashbaev A, Pritchard J, Stevens M, Flutre T, Wen X, Dermitzakis ET, Lappalainen T, Guigo R, Monlong J, Sammeth M, Koller D, Battle A, Mostafavi S, McCarthy M, Rivas M, Maller J, Rusyn I, Nobel A, Wright F, Shabalin A, Feolo M, Sharopova N, Sturcke A, Paschal J, Anderson JM, Wilder EL, Derr LK, Green ED, Struewing JP, Temple G, Volpi S, Boyer JT, Thomson EJ, Guyer MS, Ng C, Abdallah A, Colantuoni D, Insel TR, Koester SE, Little AR, Bender PK,
Lehner T, Yao Y, Compton CC, Vaught JB, Sawyer S, Lockhart NC, Demchok J, Moore HF, GTEx Consortium. The Genotype-Tissue expression (GTEx) project. Nat Genet 2013:45:580-5.

9 Sunkin SM, Ng L, Lau C, Dolbeare T, Gilbert TL, Thompson CL, Hawrylycz M, Dang C. Allen brain atlas: an integrated spatio-temporal portal for exploring the central nervous system. Nucleic Acids Res 2013;41:D996-D1008.

10 Matthijs G, Schollen E, Pardon E, Veiga-Da-Cunha M, Jaeken J, Cassiman JJ, Van Schaftingen E. Mutations in PMM2, a phosphomannomutase gene on chromosome 16p13, in carbohydrate-deficient glycoprotein type I syndrome (Jaeken syndrome). Nat Genet 1997;16:88-92.

11 Schiff M, Roda C, Monin M-L, Arion A, Barth M, Bednarek N, Bidet M, Bloch C, Boddaert N, Borgel D, Brassier A, Brice A, Bruneel A, Buissonnière R, Chabrol B, Chevalier M-C, Cormier-Daire V, De Barace C, De Maistre E, De Saint-Martin A, Dorison N, Drouin-Garraud V, Dupré T, Echenne B, Edery P, Feillet F, Fontan I, Francannet C, Labarthe F, Gitiaux C, Héron D, Hully M, Lamoureux S, Martin-Coignard D, Mignot C, Morin G, Pascreau T, Pincemaille O, Polak M, Roubertie A, ThauvinRobinet C, Toutain A, Viot G, Vuillaumier-Barrot S, Seta N, De Lonlay P. Clinical, laboratory and molecular findings and long-term follow-up data in 96 French patients with PMM2-CDG (phosphomannomutase 2-congenital disorder of glycosylation) and review of the literature. J Med Genet 2017;54:843-51.

12 DePristo MA, Banks E, Poplin R, Garimella KV, Maguire JR, Hartl C, Philippakis AA, del Angel G, Rivas MA, Hanna M, McKenna A, Fennell TJ, Kernytsky AM, Sivachenko AY, Cibulskis K, Gabriel SB, Altshuler D, Daly MJ. A framework for variation discovery and genotyping using next-generation DNA sequencing data. Nat Genet 2011;43:491-8.

13 Wang K, Li M, Hakonarson H. ANNOVAR: functional annotation of genetic variants from high-throughput sequencing data. Nucleic Acids Res 2010;38:e164.

14 Auton A, Brooks LD, Durbin RM, Garrison EP, Kang HM, Korbel JO, Marchini JL, McCarthy S, McVean GA, Abecasis GR, Altshuler DM, 1000 Genomes Project Consortium. A global reference for human genetic variation. Nature 2015;526:68-74.

15 Auer PL, Reiner AP, Wang G, Kang HM, Abecasis GR, Altshuler D, Bamshad MJ, Nickerson DA, Tracy RP, Rich SS, Leal SM, NHLBI GO Exome Sequencing Project. Guidelines for large-scale sequence-based complex trait association studies: lessons learned from the NHLBI exome sequencing project. Am J Hum Genet 2016;99:791-801.

16 Lek M, Karczewski KJ, Minikel EV, Samocha KE, Banks E, Fennell T, O'Donnell-Luria AH, Ware JS, Hill AJ, Cummings BB, Tukiainen T, Birnbaum DP, Kosmicki JA, Duncan LE, Estrada K, Zhao F, Zou J, Pierce-Hoffman E, Berghout J, Cooper DN, Deflaux N, DePristo M, Do R, Flannick J, Fromer M, Gauthier L, Goldstein J, Gupta N, Howrigan D, Kiezun A, Kurki MI, Moonshine AL, Natarajan P, Orozco L, Peloso GM, Poplin R, Rivas MA, Ruano-Rubio V, Rose SA, Ruderfer DM, Shakir K, Stenson PD, Stevens C, Thomas BP, Tiao G, Tusie-Luna MT, Weisburd B, Won H-H, Yu D, Altshuler DM, Ardissino D, Boehnke M, Danesh J, Donnelly S, Elosua R, Florez JC, Gabriel SB, Getz G, Glatt SJ, Hultman CM, Kathiresan S, Laakso M, McCarroll S, McCarthy MI, McGovern D, McPherson R, Neale BM, Palotie A, Purcell SM, Saleheen D, Scharf JM, Sklar P, Sullivan PF, Tuomilehto J, Tsuang MT, Watkins HC, Wilson JG, Daly MJ, MacArthur DG, Exome Aggregation Consortium. Analysis of protein-coding genetic variation in 60,706 humans. Nature 2016;536:285-91.

17 Gilissen C, Hehir-Kwa JY, Thung DT, van de Vorst M, van Bon BWM, Willemsen MH, Kwint M, Janssen IM, Hoischen A, Schenck A, Leach R, Klein R, Tearle R, Bo T, Pfundt $R$, Yntema HG, de Vries BBA, Kleefstra T, Brunner HG, Vissers LELM, Veltman JA. Genome sequencing identifies major causes of severe intellectual disability. Nature 2014:511:344-7.

18 Dubuc G, Tremblay M, Paré G, Jacques H, Hamelin J, Benjannet S, Boulet L, Genest J, Bernier L, Seidah NG, Davignon J. A new method for measurement of total plasma PCSK9: clinical applications. J Lipid Res 2010;51:140-9.

19 Paquette M, Luna Saavedra YG, Chamberland A, Prat A, Christensen DL, LajeunesseTrempe F, Kaduka L, Seidah NG, Dufour R, Baass A. Association between plasma proprotein convertase subtilisin/kexin type 9 and the presence of metabolic syndrome in a predominantly rural-based sub-Saharan African population. Metab Syndr Relat Disord 2017;15:423-9.

20 Ly K, Essalmani R, Desjardins R, Seidah NG, Day R. An unbiased mass spectrometry approach identifies glypican-3 as an interactor of proprotein convertase subtilisin/ kexin type 9 (PCSK9) and low density lipoprotein receptor (LDLR) in hepatocellular carcinoma cells. J Biol Chem 2016:291:24676-87.

21 Yue P, Averna M, Lin X, Schonfeld G. The c.43_44insCTG variation in PCSK9 is associated with low plasma LDL-cholesterol in a Caucasian population. Hum Mutat 2006;27:460-6.

22 Cohen JC, Boerwinkle E, Mosley TH, Hobbs HH. Sequence variations in PCSK9, low LDL, and protection against coronary heart disease. N Eng/ J Med 2006;354:1264-72.

23 Qiu C, Zeng P, Li X, Zhang Z, Pan B, Peng ZYF, Li Y, Ma Y, Leng Y, Chen R. What is the impact of PCSK9 rs505151 and rs11591147 polymorphisms on serum lipids level and cardiovascular risk: a meta-analysis. Lipids Health Dis 2017;16:1-13.

24 Le Bizec C, Vuillaumier-Barrot S, Barnier A, Dupré T, Durand G, Seta N. A new insight into PMM2 mutations in the French population. Hum Mutat 2005;25:504-5.

25 Poirier S, Mayer G, Poupon V, McPherson PS, Desjardins R, Ly K, Asselin M-C, Day R, Duclos FJ, Witmer M, Parker R, Prat A, Seidah NG. Dissection of the endogenous cellular pathways of PCSK9-induced low density lipoprotein receptor degradation: evidence for an intracellular route. J Biol Chem 2009;284:28856-64. 
26 Cameron J, Holla Øystein L, Ranheim T, Kulseth MA, Berge KE, Leren TP. Effect of mutations in the PCSK9 gene on the cell surface LDL receptors. Hum Mol Genet 2006;15:1551-8.

27 Benjannet S, Rhainds D, Hamelin J, Nassoury N, Seidah NG. The proprotein convertase (PC) PCSK9 is inactivated by furin and/or PC5/6A: functional consequences of natural mutations and post-translational modifications. J Biol Chem 2006;281:30561-72.

28 Susan-Resiga D, Girard E, Kiss RS, Essalmani R, Hamelin J, Asselin M-C, Awan Z, Butkinaree C, Fleury A, Soldera A, Dory YL, Baass A, Seidah NG. The proprotein convertase subtilisin/kexin type 9-resistant R410S low density lipoprotein receptor mutation: a novel mechanism causing familial hypercholesterolemia. J Bio/ Chem 2017;292:1573-90.

29 Seidah NG, Awan Z, Chrétien M, Mbikay M. Pcsk9: a key modulator of cardiovascular health. Circ Res 2014;114:1022-36.

30 Van Schaftingen $E$, Jaeken J. Phosphomannomutase deficiency is a cause of carbohydrate-deficient glycoprotein syndrome type I. FEBS Lett 1995;377:318-20.

31 Westphal V, Peterson S, Patterson M, Tournay A, Blumenthal A, Treacy EP, Freeze HH. Functional significance of PMM2 mutations in mildly affected patients with congenital disorders of glycosylation la. Genet Med 2001;3:393-8.

32 Rao AS, Lindholm D, Rivas MA, Knowles JW, Montgomery SB, Ingelsson E. Large-Scale Phenome-Wide Association Study of PCSK9 Variants Demonstrates Protection Against Ischemic Stroke. Circ Genom Precis Med 2018;11:e002162.

33 Harvey PD, Sabbagh MN, Harrison JE, Ginsberg HN, Chapman MJ, Manvelian G, Moryusef A, Mandel J, Farnier M. No evidence of neurocognitive adverse events associated with alirocumab treatment in 3340 patients from 14 randomized phase 2 and 3 controlled trials: a meta-analysis of individual patient data. Eur Heart $J$ 2018;39:374-81.

34 Mannarino MR, Sahebkar A, Bianconi V, Serban M-C, Banach M, Pirro M. Pcsk9 and neurocognitive function: should it be still an issue after Fourier and EBBINGHAUS results? J Clin Lipido/ 2018;12:1123-32.

35 Seidah NG, Chrétien M, Mbikay M. The ever-expanding SAGA of the proprotein convertases and their roles in body homeostasis: emphasis on novel proprotein convertase subtilisin kexin number 9 functions and regulation. Curr Opin Lipidol 2018;29:144-50.

36 Schedin-Weiss S, Winblad B, Tjernberg LO. The role of protein glycosylation in Alzheimer disease. Febs J 2014;281:46-62.

37 Herber J, Njavro J, Feederle R, Schepers U, Müller UC, Bräse S, Müller SA, Lichtenthaler SF. Click Chemistry-mediated biotinylation reveals a function for the protease BACE1 in modulating the neuronal surface glycoproteome. Mol Cell Proteomics 2018;17:1487-501.

38 Liang H-C, Russell C, Mitra V, Chung R, Hye A, Bazenet C, Lovestone S, Pike I, Ward M. Glycosylation of human plasma clusterin yields a novel candidate biomarker of Alzheimer's disease. J Proteome Res 2015;14:5063-76.

$39 \mathrm{Scott} \mathrm{H}$, Panin VM. N-Glycosylation in regulation of the nervous system. Adv Neurobiol;9:367-94. 